The final publication is available at Elsevier via http://dx.doi.org/10.1016/j.chroma.2017.01.007 (C) 2017. This manuscript version is made available under the CC-BY-NC-ND 4.0 license http://creativecommons.org/licenses/by-nc-nd/4.0/

\title{
Development of a Multichannel Microfluidic System with Schlieren Imaging Microscopy for Online Chip-Based Moving Boundary Electrophoresis
}

Atefeh S. Zarabadi ${ }^{1}$, Janusz Pawliszyn ${ }^{* 1}$, Mojtaba Hajialamdari ${ }^{2}$

${ }^{1}$ Department of Chemistry, University of Waterloo, Waterloo, Ontario, Canada N2l 3G1

${ }^{2}$ Department of Physics and Astronomy, University of Waterloo, Waterloo, Ontario, Canada N2l 3G1

*E-mail: janusz@uwaterloo.ca Phone: 519-888-4567 x35123

\begin{abstract}
The concentration gradient detection method based on the Schlieren optics employed for electrophoresis analyses by extending the technology to a multi-channel system using a prototyped microfluidic chip (thinXXS Micro-technology, Germany). The results prove that coupling a chip-based microfluidic device with Schlieren detection is an appropriate approach to improve the electrophoretic separations. The effects of channel's geometry and dimension were investigated by conducting the experiments in channels with different cross sectional areas. Fast kinetic data acquisition of the charge-coupled device (CCD) camera facilitated recording of a time sequence of optical images, demonstrating the potential of the CCD camera as a powerful tool for studying dynamic processes such as diffusion. Diffusion coefficients of sample proteins were measured under static and dynamic conditions, where the static mode demonstrated more accurate results. Furthermore, the Fourier transformation was employed to improve the Schlieren images for quantitative analysis of the diffusion coefficient measurement.
\end{abstract}

\section{Keywords}

Capillary isoelectric focusing; concentration gradient detection; moving boundary electrophoresis; multi-channel chip; Schlieren imaging microscopy. 


\section{Introduction}

Schlieren optics provides a powerful technique for visualizing changes or non-uniformities in the refractive index of transparent media [1]. It has been used for over two centuries, since the early 1800s. Schlieren systems have been typically implemented for a wide variety of real-time fluid dynamics studies [2]. Widespread availability of digital imaging systems allows Schlieren optics to be applied to the study of transparent media in novel ways. Application of Schlieren detection in analytical chemistry has been comprehensively reviewed in the literature [3,4]. This detection method was found to be particularly sensitive with those CE modes that show self-concentration and focusing effects, such as capillary isoelectric focusing (CIEF) [5-9]. Although the self focusing effect is not present in moving boundary electrophoresis (MBE), the Schlieren technique was successfully employed [10,11], because of the high concentration gradient generated in the boundary between samples and buffer. A multipurpose capillary electrophoresis system demonstrates that all four separation modes can be coupled with concentration gradient detection using Schlieren as a universal detector [12]. The concentration gradient detection method based on Schlieren optics offers advantages such as sensitivity and enhanced resolution in addition to drift reduction [3]. In spite of the unique advantages offered by this technique, its application in analytical chemistry in previous studies shows some drawbacks regarding sample throughput, and quantitative analysis.

The analytical signals can be proportional to the average of the analyte concentration that reflects the true concentration value (C), for example in absorbance detection. The signal amplitude can also depend on the non-uniform distribution of analytes in the detection volume as in gradient-based detectors, which results in the derivative form of concentration $(\mathrm{dC} / \mathrm{dx})$. The latter type of method ignores the drifts associated with the slow variations in the concentration by emphasizing the changes in the concentration, which makes it an appropriate choice for Fourier transform analysis. The derivative nature of the concentration gradient allows effective removal of the refractive index drifts produced by temperature fluctuation. 
The application of concentration gradient detection to the determination of diffusion coefficients has been already performed; however, its quantitative analysis depends on conventional peak height measurements and needs to be improved [13]. The present study explores the data analysis of Schlieren images, by calculating the diffusion coefficient in the frequency domain. The Fourier transformation approach is the same as what has been employed for analysis of the diffusion data from capillary isoelectric focusing with whole column imaging detection [14].

\section{Method and materials}

All optical elements and posts were bought from ThorLabs, USA. The chemicals were of analyticalreagent grade, and purchased from Sigma Aldrich. The solutions were prepared using sonicated ultrapure water (18 M $\Omega$ ) from a Barnstead/Thermolyne system. The samples were filtered with a $0.2 \mu \mathrm{m}$ pore-size membrane.

\subsection{Schlieren Set-up}

The system was mounted on a vibration isolation table. The components were arranged as outlined in the supplementary information (Figure S1). The design of a Schlieren system suggests a compromise between sensitivity, image resolution, and the size of the field-of-view. The required sensitivity should accordingly be estimated by the employed optical system; it is proportional to the focal length of the collecting lens and inversely proportional to the Stop size. Thus, a small blocking Stop and lens with large focal length provide high sensitivity. In this study the Stop size is $500 \mu \mathrm{m}$. The system implemented a beam-expander lens to expand the green laser $(532 \mathrm{~nm}$, power $\leq 5 \mathrm{~mW})$ beam to a $5 \mathrm{~cm}$ diameter light spot, and was equipped with filters and a pinhole to adjust to a desired spot diameter and lower the power. The collimated light traversed another lens (L1) to reach the test area, where the chip was located. Depending on the experiment, the light was deflected by or straightly passed through the Schlieren lens (L2); those rays that were not deflected would focus at the Stop and were blocked there, while the rays that were deflected by the sample inside the channel changed their path and reached the camera. The deflected beams were focused and hit the sensor via the image transfer lens (L3). The test region was delimited by the space between L1 and L2, where the multi-channel chip was loaded on a 2D-stage (Figure S2). The chip was held firmly in place after putting the desired channels in the center 
of the light beam by tilting the slide in xy-plane. After adjusting the chip into the right position, the electrode comb was mounted on its top.

\subsection{Chip Specifications and Configurations}

A prototype multi-channel chip in a miniaturized cyclic olefin copolymer (COC) microfluidic device (thinXXS Micro-technology, Germany) was employed for protein analysis (Figure S3). The short channels, $18 \mathrm{~mm}$, of the introduced chip were embedded in a slide with a footprint of $7.5 \times 2.5\left(\mathrm{~cm}^{2}\right)$. The cyclo-olefin copolymer combines excellent optical properties with biocompatibility and good chemical resistance to most acids and bases. The linear channel slide features 5 straight channels of different cross sectional areas. The COC-chip design, with its short channels and integrated reservoirs, suggests a simple configuration for robust analysis, with moving boundary electrophoresis. The standard ports with a capacity of $25 \mu \mathrm{L}$ were used as reservoirs in the MBE experiment. To perform the CIEF experiment, larger reservoirs, injection capillaries, and membranes were incorporated into the prototyped chip. For the multi-channel to function, an electric field needs to be generated through each channel. This was achieved using an electrode comb, which distributes the voltage to each channel through a separate electrode pair. A photo-mask sheet was aligned to the channels and faced the laser source to avoid stray light.

\subsection{CE Techniques}

The multi-channel chip was primarily tested with capillary isoelectric focusing for separation of myoglobin isoforms with UV whole column imaging detection (WCID). Further experiments were performed in moving boundary electrophoresis mode, and the signal was detected by Schlieren optics inside multiple channels. With both separation techniques, diffusion coefficients were estimated in two modes that can be described as static and dynamic, respectively, depending on the fluid motion at the time of diffusion measurement. Imaged CIEF was performed on an iCE280 Analyzer (Convergent Bioscience [now Protein Simple], Toronto, Canada), inside a commercial separation cartridge. In the MBE, the sample was introduced through emptying and then filling the reservoir at the anodic end with a sample solution. This step was accomplished using injection syringes and a small diameter plastic tube to evacuate the anodic reservoir. The sample boundary was driven by applying a $500 \mathrm{~V}$ potential difference from a high-voltage D.C. power supply. The empty/fill mechanism is depicted in Figure S2. 


\subsection{Analysis of The Schlieren Images}

The employed CCD camera (Model: DR32400-FI, Andor, USA) is a silicon-based semiconductor chip bearing a two-dimensional matrix of photo-sensors, or pixels. This matrix has a pixel array for an imaging area of $26.4 \mathrm{~mm} \times 3.2 \mathrm{~mm}$. SOLIS proprietary software was used to control the camera and analyze the time-resolved imaging efficient data acquisition and processing. Full resolution image readout mode provided a 3D-image of $1650 \times 200$ pixels, and the z-direction showed the amplitude of the signal, which was determined by the intensity of the deflected light that reached the camera sensor. With the selected optical elements, the CCD could monitor the effective length of $3.5 \mathrm{~cm}$ that covered 5 channels. However to minimize the effect of uneven distribution of the laser light, it was restricted to partial channel imaging detection by the width of the sensor at the center of the beam spot for simultaneous monitoring of two channels.

The CCD camera can acquire a complete scan in microseconds, which allows for rapid kinetic measurements. The kinetics acquisition mode was employed to record the dynamic process; this mode is particularly well suited to recording the temporal evolution of a process such as diffusion. To enhance the quality of the images, signals were processed through background correction, normalization, and noise reduction. Without these corrections, the images included additional noise and baseline fluctuations, which would have made the quantitative analysis difficult. Data treatment was performed using the Andor BASIC programming language included with SOLIS. The amplitude of the photons detected in an image was obtained by subtracting the total photon count of the background image from each image frame in the kinetic series, and displayed as background corrected count unit. Background is a data acquisition made in darkness. It is made up of fixed pattern noise, and any signal due to dark current. Correction for the background Count, also improves the signal-to-noise ratio of the image. The signal-to-noise ratio was further improved by increasing the number of accumulations, in which data from several scans of the same image were recorded and averaged by the fast scanning CCD sensor to reduce noise. This results in improved SNR, at the cost of temporal resolution loss. However, the required temporal resolution is obtained after accumulation by the fast scanning rate offered by the camera. The camera temperature was set to zero centigrade and maintained at this temperature during the experiment to minimize the shot noise. 


\section{Results and Discussion}

The capillary isoelectric focusing experiments were performed by UV-vis whole column imaging detection in channel $\mathrm{C}$ of the microchip as an identical separation column to the capillary. The results provide insight about the chip performance compared to the capillary cartridge. Table S-1 summarized some of the key features of the multi-channel chip and compared them to the commercial capillary cartridge. A straightforward advantage of using microchip in CIEF-WCID is the absence of lens effect, which contributes to better detection sensitivity.

The COC multi-channel chip was examined by CIEF-UV-WCID; different parameters such as reproducibility, resolution, and wall-interaction were compared to those of the commercial capillary cartridge. A comparison of the separation experiments in the COC-chip and the commercial chip, as demonstrated in Figure S-4, showed that the myoglobin isoforms were fairly well resolved in both devices. The light intensity in the COC-chip is higher than that of capillary cartridge due to the lens effect. As a result, the peak heights of both myoglobin isoforms in square channel COC-chip are larger than the one measured in round capillary cartridge. Due to the inert surface of the plastic chip, its channel preconditioning for EOF suppression was not as important, compared to the fused silica in the commercial cartridge. A relative standard deviation of $1.8 \%$ for determination of the peak positions in three trials showed lower but comparable reproducibility to the similar experiment in the commercial channel.

One of the apparent limitations of using round capillaries for path-length-dependent detection techniques such as UV absorbance is called lens effect. As shown in Figure S-5, the light intensity profiles of a water-filled round capillary and a rectangular channel, experimentally confirmed that the light beam passing a microchip have higher intensity than a capillary. The intensity of an incident light passing through a capillary of $100 \mu \mathrm{m}$ ID was decreased to nearly one third of that passing through micro-channel of $100 \mu \mathrm{m} \times 100 \mu \mathrm{m}$.

In moving boundary electrophoresis as a principal mode of CE, the samples are introduced through the frontal injection method into the capillary without discrimination. It provides higher sensitivity compared to the zone technique because no further dilution happen during the separation process. This approach, in combination with the Schlieren system has been already utilized for separation and detection of amino acids and carbohydrates [16]. However, the aim of this research is determination of 
the diffusion coefficient of proteins, and as such, the separation is not of main concern. Effect of channel dimension, as the detection cell, on detection sensitivity were examined by passing green laser light through different channels of the COC-chip and detecting by Schlieren imaging system.

Effect of dimension specification of the separation column is particularly important in optical detection technologies, as it does not only affect the separation efficiency but also influences the sensitivity of the method, as a detection cell. The employed COC chip consists of multiple channels with different crosssections in square and rectangular forms. Pressure-driven flows in the channels can lead to large dispersion, which is because of lower convective velocity of the solute near the walls than it is in the center. Accordingly, among the eight channels on the chip, four are too wide for the purpose of the experiment, and have very fast hydrodynamic flow rates. Thus, to investigate the effect of channel geometry and dimension, the experiments were conducted in the other four channels, with specifications shown in Table S-2. Two of the channels, labeled D, have exactly the same dimensions, hence they are employed in simultaneous measurements and verifying reproducibility of the experiments. The diameter of the non-circular channels is defined as hydraulic diameter, $\mathrm{DH}$, which is reciprocal to the diameter of a round tube or capillary, making for an easier comparison.

Channel $\mathrm{C}$ is the deepest, hence providing the longest light path. The width of channel $\mathrm{D}$ is half of others hence considered the narrowest channel. Channel E is rather planar, with a shallow depth and wide breadth. Different dimensions make different surface-area-to-volume ratios (SVR) for each channel. The heat is dissipated more efficiently when the SVR is higher.

Table 1 Diffusion coefficient measurement of tryptophan (1 $\mathrm{mg} / \mathrm{ml})$ by moving boundary electrophoresis coupled with Schlieren imaging detection within different channels.

\begin{tabular}{clll}
\hline Channel & $\mathrm{C}$ & $\mathrm{D}$ & $\mathrm{E}$ \\
\hline $\begin{array}{c}\mathrm{D}_{\text {tryp. }} \\
\left(\mathrm{cm}^{2} / \mathrm{s}\right)\end{array}$ & 6.9 & 6.8 & 7.0 \\
$\begin{array}{c}\text { \%Error } \\
\text { a }\end{array}$ & 5.3 & 3.8 & 6.8 \\
\%RSD $^{\mathrm{b}}$ & 6.8 & 4.4 & 7.1
\end{tabular}

a. Error percent is calculated based on the reported diffusion coefficient in the literature. [17]

b. Relative standard deviation is determined in percent for three trials $(\mathrm{n}=3)$. 
The experiments were conducted for three replicates and the RSD values demonstrate reproducibility of the diffusion coefficient measurements in each channel (Table 1). Channel C showed the least precision in measurements due to the higher influence of the hydrodynamic flow in the presence of subtle differences in the electrolyte levels. The experiment in channels $\mathrm{C}$ and $\mathrm{E}$ showed a higher error margin compared to channel D. The diffusion coefficient estimation in channel E with binning method deviated the most from the true value, as the number of rows with poor SNR are doubled compared to channel D. In general, all channels have acceptable results and the effect of dimension is not very considerable. The dimension factors that slightly influenced the measurement are the shallower depth of the channels that resulted in higher sensitivity due to lower stray light, and the breadth of the channels, which inversely affected the precision and accuracy of the measurements because of increasing the dispersion and lowering the SNR. Channel D with square cross-section, $\mathrm{DH}=50 \mathrm{~m}$, and highest surface-to-volume ratio $\left(0.08 \mathrm{M}^{-1}\right)$ proved to have the most suitable dimensions for this experiment.

Figure 1 Schlieren 3D-image recorded 45s after applying the voltage; x-pixel number, y-rows, and z-counts (background corrected), for estimation of the tryptophan diffusion coefficient by MBE experiment simultaneously in two D channels.

Figure 1 illustrates the MBE of tryptophan in two D channels simultaneously; the image is recorded after 45 seconds of applying voltage. The relative standard deviation for three trials in channels D indicates acceptable reproducibility of $4.4 \%$. However, the COC chip precision in the CIEF experiment (1.8\%) was much better than that of MBE, due to different sample introduction and experimental procedures. Reproducibility in CE methodologies can be compromised by different factors including buffers, capillary, modes of injections, and applied voltage [18]. The major source of experimental variation in moving boundary electrophoresis was the non-automated injection system; the empty/fill 
step was the most vulnerable stage of the experiment, in which there might be inconsistencies due to incomplete withdrawal of the electrolyte. The reproducibility of the experiment can be improved by replacing the manual sample introduction with an automated one. Although the samples were injected parallel into the channels, the image shows that they are not moving together, this might be due to same reasons arise irreproducibility.

Figure 2 Schlieren images of tryptophan's moving boundary electrophoresis and its dispersion illustrated in four time frames. The corresponding movie is provided in the supplementary information.

The raw images of tryptophan MBE are demonstrated in Figure 2, where the dispersion and immigration of the sample boundary is depicted as a broadening bright spot.

\subsection{Determination of diffusion coefficient}

Microfluidic devices can be used in a variety of interesting applications including molecular diffusion coefficient measurement [19]. Diffusion coefficient measurements by dynamic method of MBE with imaging Schlieren detection is compared with static imaging method of CIEF with UV-WCID inside channel $\mathrm{C}$ of the micro-fabricated multi-channel device. These comparisons were made using tryptophan and BSA samples. The evaluation of the precision and accuracy of the measurements proved better performance of static mode. However, either of the methods had its own advantages and shortcomings; the diffusion coefficient measurement by the static method (CIEF) was more accurate, and robust, whereas MBE method was simpler and faster with acceptable results within experimental 
error range. In the literature, estimation of diffusion coefficients has been suggested based on signal peak height for the CIEF with a concentration gradient Schlieren imaging detector [13].

As shown by experimental results, the calculation in the time domain, especially based on peak height and width, is not as effective as the frequency domain [14]. An average concentration detector would visualize the moving boundary signal as a sigmoid peak, which is not easy to deal with in the FT approach, however, using the concentration gradient detection (Schlieren); the output signal would have a derivative form that looks like a Gaussian-wise shape. Hence, in the current research, the diffusion coefficient measurements were calculated by Fourier transformation method.

Table 2 Diffusion coefficient measurements by two approaches; Dynamic method, D Dyn, through MBE with Schlieren imaging detection, and Static method, D Stc, through CIEF experiment followed by UVWCID.

\begin{tabular}{|c|c|c|c|c|c|c|}
\hline Amino acid/ Protein & $\begin{array}{c}\text { D stc. } \\
\left(\mathrm{cm}^{2} / \mathrm{s}\right)\end{array}$ & $\begin{array}{c}\text { D Dyn. } \\
\left(\mathrm{cm}^{2} / \mathrm{s}\right)\end{array}$ & \multicolumn{2}{|c|}{$\begin{array}{c}\text { D Lit. } \\
\left(\mathrm{cm}^{2} / \mathrm{s}\right)\end{array}$} & $\begin{array}{l}\text { \%Error } \\
\text { Stc. }\end{array}$ & $\begin{array}{l}\text { \%Error } \\
\text { Dyn. }\end{array}$ \\
\hline Tryptophan & $\begin{array}{l}6.2 \times 10^{-} \\
6\end{array}$ & $\begin{array}{l}6.9 \times 10^{-} \\
6\end{array}$ & $\begin{array}{l}6.55 \\
{[17]}\end{array}$ & $\times 10^{-6}$ & 5.3 & 7.0 \\
\hline Bovine Serum Albumin & $\begin{array}{l}5.3 \times 10^{-} \\
7\end{array}$ & $\begin{array}{l}6.7 \times 10^{-} \\
7\end{array}$ & $\begin{array}{l}5.90 \\
{[20]}\end{array}$ & $\times 10^{-7}$ & 10.1 & 14.1 \\
\hline
\end{tabular}

After binning the channel rows in the image at different dispersion time intervals, the obtained profiles were used for determination of diffusion coefficient through FT approach. The Fourier analysis was applied directly to the signal. Tryptophan and BSA diffusion coefficients were determined in channel C with error percent $14.1 \%$ and $7.0 \%$ respectively. The diffusion coefficient was measured in a static fashion in the CIEF experiment, where the focused protein band started to diffuse after the voltage was disconnected and the sample plug moves due to the concentration gradient with its vicinity media. However, in the MBE with Schlieren detection, the measurements were performed in dynamic mode, in which the diffusion of the sample front was monitored in presence of the electric field, an on-the-flyelectrophoresis. The former mode is inherently more appropriate for measuring the diffusion coefficient. Thus, the comparison of the obtained values (Table 2) by either of these two methods reinforced the higher efficiency of the static mode for this purpose. 


\subsection{Limitations of the technique}

Beside advantages offered by concentration gradient Schlieren imaging detection, there are some drawbacks associated with both the concentration gradient technique and the operational Schlieren optics, which affect the efficiency of the method. Refractive index gradient measurement is a universal technique.

The main advantage of this inherent feature of the refractive index gradient detector is that a wide variety of compounds can be detected by this method. However, this universal feature results in the contribution of other existing compounds, such as carrier ampholytes in CIEF experiments, to the signal and makes it noisy and inappropriate for quantitative analysis. On the other hand, increasing the solute concentration to enhance the difference between the refractive index of the analyte and that of the solvent is not suitable for determination of diffusion coefficient, which is more accurately estimated in dilute solutions.

The most obvious instrumental limitation of the studied Schlieren system is the size and portability. The size of the system depends on the size and focal length of the employed lenses. All the systems elements are fixed on a vibration isolation table in a dark room to avoid any additional ambient noise. Hence, the current system is neither flexible nor portable. Automation is another critical issue in CE experiments since manual injection and optimization of the optical elements and Stop for each trial is tedious tasks, which also affect the repeatability of the operation that is necessary for precise quantitative analysis. The quality of the employed optical elements plays an important role in the sensitivity and resolution of the method.

\section{Conclusions}

The miniaturized multi-channel chip was successfully coupled with concentration gradient detection based on Schlieren optics and demonstrated a great potential for universal, sensitive, and highthroughput measurements of diffusion coefficients of proteins. The effect of channel geometry and dimension on the separation and detection was explored with respect to the light path as well as wallinteraction and dispersion of the solute. This evaluation proves that the proposed multi-channel chip is a viable choice for electrophoresis experiments. The square or rectangular micro-channel eliminates the lens effect drawback associated with round capillaries. The effective compatibility of the Schlieren imaging system with chip-based microfluidic device makes it an appropriate choice for electrophoresis 
analysis. Using multiple channels for MBE experiment can greatly increase the sample throughput, making it comparable with gel slab electrophoresis, with much faster analysis speed in a channel. The speed and high resolution of the CCD camera make it an attractive choice for exploring dynamic and kinetic processes. Further enhancement of the imaging system is still possible, by improving the optical elements. In summary, the proposed experimental method can be employed for simple and fast analysis of proteins to accurately determine their diffusion coefficient value.

\section{Supporting Information}

The Supporting Information documents are available online.

\section{References}

[1] G.S. Settles, Schlieren and Shadowgraph Techniques: Visualizing Phenomena in Transparent Media., 2nd ed., Springer, 2006.

[2] G.S. Settles, G. S. Settles, Schlieren and Shadowgraph Techniques, in: Large-F. and Focusing Schlieren Methods, Springer-Verlag Berlin Heidelberg, 2001.

[3] J. Pawliszyn, Concentration Gradient Detection Based on Sclieren Optics, Spectrochim. Acta Rev. 13 (1990) 311-354.

[4] L. Vonguyen, J. Wu, J. Pawliszyn, Peptide mapping of bovine and chicken cytochrome c by capillary isoelectric focusing with universal concentration gradient imaging., J. Chromatogr. B. Biomed. Appl. 657 (1994) 333-8.

[5] J. Wu, P. Frank, J. Pawliszyn, Diode Laser-Based Concentration Gradient Detector for Detection of Capillary Isoelectric Focusing, Appl. Spectrosc. 46 (1992) 1837-40.

[6] J. Wu, J. Pawliszyn, Application of capillary isoelectric focusing with universal concentration gradient detector to the analysis of protein samples., J. Chromatogr. 608 (1992) 121-30.

[7] J. Wu, J. Pawllszyn, Dual Detection for Capillary Isoelectric Focusing with Refractive Index Gradient and Absorption Imaging Detectors, Anal. Chem. 66 (1994) 867-873.

[8] T. McDonnell, J. Pawliszyn, Capillary isotachophoresis with concentration-gradient detection An application to the separation of synthetic peptides, J. Chromatogr. 559 (1991) 489-97. 
[9] T. McDonnell, J. Pawliszyn, Capillary isotachophoresis with concentration gradient detection., Anal. Chem. 63 (1991) 1884-9.

[10] J. Pawliszyn, Concentration Gradient Detector in Capillary Separation Technology, J. Liq. Chromatogr. 10 (1987) 3377-3392.

[11] J. Pawliszyn, J. Wu, Moving boundary capillary electrophoresis with concentration gradient detection, J. Chromatogr. 559 (1991) 111-118.

[12] J. Pawliszyn, J. Wu, Multi-Purpose Capillary Electrophoresis System With Concentration Gradient Detection, Talanta. 39 (1992) 1281-1288.

[13] J. Wu, J. Pawliszyn, Capillary isoelectric focusing with a universal concentration gradient imaging system using a charge-coupled photodiode array, Anal. Chem. 64 (1992) 219-224.

[14] A.S. Zarabadi, J. Pawliszyn, Accurate Determination of the Diffusion Coefficient of Proteins by Fourier Analysis with Whole Column Imaging Detection, Anal. Chem. 87 (2015) 2100-2106.

[15] T. Tsuda, J. V. Sweedler, R.N. Zare, Rectangular capillaries for capillary zone electrophoresis, Anal. Chem. 62 (1990) 2149-2152.

[16] J. Pawliszyn, J. Wu, Moving boundary capillary electrophoresis with concentration gradient detection, J. Chromatogr. 559 (1991) 111-118.

[17] F. Ye, H. Jensen, S.W. Larsen, A. Yaghmur, C. Larsen, J. Østergaard, Measurement of drug diffusivities in pharmaceutical solvents using Taylor dispersion analysis., J. Pharm. Biomed. Anal. 61 (2012) 176-83.

[18] I. Ali, H.Y. AboulEnein, V.K. Gupta, Precision in Capillary Electrophoresis, Anal. Lett. 39 (2006) 2345-2357.

[19] A.E. Kamholz, B.H. Weigl, B. a. Finlayson, P. Yager, Quantitative analysis of molecular interaction in a microfluidic channel: The T-sensor, Anal. Chem. 71 (1999) 5340-5347.

[20] Z. Liu, T. Lemma, J. Pawliszyn, Capillary Isoelectric Focusing Coupled with Dynamic Imaging Detection: A One-Dimensional Separation for Two-Dimensional Protein Characterization, J. Proteome Res. 5 (2006) 1246-1251. 